\title{
EXPERIENCIAS DE AULA
}

UNA PROPOSTA ALTERNATIVA DE MODIFICACIÓ EN L'ENSENYAMENT DE LA QUÍMICA. DISSOLUCIONS

\section{Carbó Aguilar, S. Usó Mañanós, J.}

IB Ramon Llull, València.

IB Francesc Tarrega, Vila-real, Castelló

\section{Introducció}

Entre els models d'aprenentatge més emprats actualmentes trobenels d'Ausubel, Piaget, Gagné iels inspirats en la Psicologia del Processament de Ia Informació (Gutierrez 1989). Fn aquest treball d'aplicació, utilitzarem la teoria de l'estructura del coneixement cognoscitiu de Piaget $(1972,1981)$ com a punt de partença, puix pensem que qualsevol modificació del curriculum ha de considerar necessàriament el desenrotliament cognoscitiu de I'alumne; sense oblidar, evidentment, tots els altres factors (motivació, interès, conejxements previs...) que influeixen on l'aprenentatge.

El model que hem assumit ha cstat bàsicament el proposat per Gimeno Sacristán, aui l'anomena model comprensin (Gimeno 1981), el qual es configura mitjancant dos subsistemes: el psicologic -per a nosaltres, el model de Piaget-i el d'ensenyament 0 model didàctic. A més, hem adoptat I'anomenada pedagogia per «redescobrimentorientat» (Cil 1982,1983) - encara que I'autor avui parla més aviat de l'aprenentatge com una uconstruccíos (Gil i Martínez 1987)-que ens permetraे de concretar els diferents elements del model djdàctic triat.

Per a nosaltres, fer un disseny bàsic de programació que possibilite una eficaç acció docent és compatible amb l'anàlisi dels coneixements previs i preconceptes dels alumnes -per a Ausubel, $d$ 'una importància fonamental (Ausubel 1978)-, i també, amb el canvi metodològic i conceptual postulat per alguns autors (Carrascosa i Gil 1985) i amb el desenvolupament del pensament divergent especialment pel que fa a l'emissió d'hipòtesis $i$ al disseny d'experiències (Cill 1983).

\section{Disseny de treball}

L'aplicactó realitzada correspon al tema de Dissolucions, actualment inclos al programa oficial de l'assignatura rísica i Química de segon de BUP.

A partir d'uns continguts que considerem mínims, la nostra tasca ha estat d'establir els objectius que cal assolir i de dissenyar una sèrie d'activitats i d'experiencies senzilles per tal d'aconseguir-los. Alhora, hom ha relacionat indicant-ho explícitament, entre parèntesi, després de cada experiència les diferents activitats amb l'estadi de desenvolipament cognoscitiu dels alumnes, utilitzant unes taxonomies especialment dissenyades per a les ciències (Shayer i Adey 1984) i fonamentades en els treballs de Piaget.

Les activitats descrites representen un disseny curricular bàsic, una proposta oberta dotada de la suficient flexibilitat que perme:a als docents una revisió, modifjcacio i tria. Altres elements del model didàctic assumit no tractats, com ara l'avaluació, i el seguiment de la nostra proposta a l'aula, els deixem per a una anàlisi postetrior.

\section{Activitats}

A.1. Mescleu llimadures de ferro amb pols de sofre.. Penseu algun mètode per separar-los tots dos. Després de realitzar-ho, indiqueu si el mètode utilitzat ês físjc o químis. $(2 \mathrm{~B} / 3 \mathrm{~A})$.

A.2. Preneu aigua satada. Busqueu a1gun procediment que permeta extreure'n la sal. En aplicar-lo, indiqueu si és un procediment .ísic o químic. (3A).

A.3. Cerquen diferències en la manera de presentat-se els components que heu barrejat en les activitats A.l i A.2. Poseu exemples de mescles i de dissolucions i comenteu-ho. (2B).

A.4. Penseu algun mètode que permeta de separar els components constituents de l'aigua destil.lada. Cal discutir les diverses propostes fetes, (3A).

A.S. En un got amb aigua heu de posar dos elèctrodes i dos tubs d'assaig (plens d'aigua), tal com s'indica en la figura 1 .
Connecteu els elèctrodes en una pila. Observeu el que passa. Cal repetir I'experiència, però afegint unes gotes d'àcid sulfúric. Heu d'analizar-la acudint, sical, a la bibliografia per interpretarla. $(3 \mathrm{~A})$.

Figuro 1

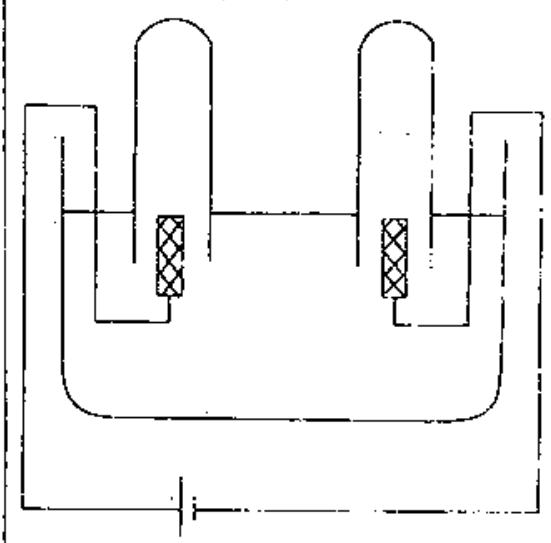

A.6. Preneu un tros de fil de coure i un tros de plom. Heu d'idear mètodes que permeten descompondre'Is. Comenteulos $i$ arribeu a conclusions. (2B/3A).

A.7. Situeu aquestes substàncies: vi, aigua amb sucre, granit, aire, ferro, alcohol, sal, acer, calç, melmelada i llautó, en algun dels quatre grups segiients: clements químies, composts, dissolucions i mescles. (2B).

A.8. Ficu un esquema on apareguen aquestes quatre formes de presentar-se la matèria, i indiqueu els trets característics i essencials de cadasctna. (2B).

A.9. Peseu 5 g de sucre i en dissoldre's en $100 \mathrm{ml} \mathrm{d}^{\prime}$ aigua torneu-los a pesar. Compareu la massa del sucre $\mathrm{i}$ la de I'aigua (prèviament calculada) ambla de la dissolució que heu preparat. (2B).

A.10. Cal buscar aplicacions quotidianes $i$ industrials de les dissolucions indicant, alhora, la importància de conèixer els seus components. (2B). 


\section{INTERCAMBIOS, COMENTARIOS}

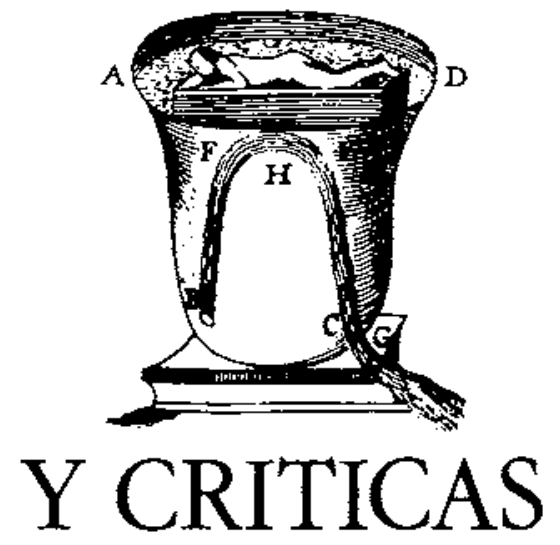

En esta sección intentamos recoger, por una parte, los comentarios y críticas sobre los trabajos aparecidos, así como sugerencias de cualquier tipo que puedan contribuir a una mejora de la revista.

En segundo lugar pretendemos que estas páginas sirvan para dar a conocer la existencia de grupos de trabajo y facilitar asi los contactos e intercambios.

También pensamos que puede ser de interés el conocimiento de las líneas de trabajo seguidas por los distintos grupos, que pueden enviar breves resumenes de sus actividades.

Por tiltimo contemplamos la posibilidad de favorecer los intercambios objeto de esta sección con la publicación de algunas entrevistas y mesas redondas.

\section{GRUPOS DE TRABAJO}

\section{SEMINARIO DE DIDÁCTICA DE LAS CIENCIAS}

Ricardo Puyol Castillo, Estanislao Camacho Dominguez

Este grupo de trabajo se encuentra adscrito en la actualidad al CEP de Málaga, comoSeminario Permanente. Está formado por seis profesores de tres centros (dos públicos y uno privado). Sus actividades están encaminadas al desarrollo de materiales curriculares para Ciencias en educación infantil y primaria.

La clección de estos niveles se ha debido a la ausencia de materiales adecuados a las características intelectuales y motivacionales de estos alumnos, y a la importancia que estas edades tienen en el desarrollo de actitudes y conocimientos que potencian el aprendizaje en etapas posteriores.
Se pretenden diseñar materiales curriculares de Geología, Biología, Física, Química y Astronomía; siempre desde una perspectiva interdisciplinar y con proyección a la educación ambiental. En este sentido, se han elaborado diversos materiales para introducir la observacion y la medida en educación primaria (Cíclo Inicial) yel conocimientodel medionatural, concretamente del mundo vegetal, en educación infantil y primaria (Preescolar y Ciclo Inicial).

Fruto de este trabajo han sido las comunicaciones realizadas: una en colaboración con el colegio Sierra Blanca de Málaga, que se ha presentado a la I Jornada de Medio Ambiente y Educación Ambiental celebrada en Mijas (Málaga, mayo de 1991); y otras dos al I Encuentro de Grupos de Trabajo del Área de Ciencias de la Naturaieza del Ámbito del CEP de Málaga; así como Ia participación en
I Concurso de Actividades de Educación Ambiental en Centros Educativos con motivo del Día Mundial deI Medio Ambiente en la Comunidad Autónoma Andaluza.

El objetivo central del seminario es la elaboración desarrollo y evaluación de materiaies curriculares para Educación Infantil y Primaria; actualmente se está en la primera etapa, y esperamos finalizar este proyecto antes de la entrada en vigor de Ia Reforma en la Enser̃anza Primaria.

Para cualquier posible contacto con el grupo:

Estanislao Camacho

Colegio «EI Romerai»

Francisco Barbín, 22

29010-Málaga 
A.11. Comenteu les dades que apareixen en aquesta taula:

Cał adonar-se de quina manera s'expressa la potabilitat d'una aigua. Contrasteu aquestes dades amb altres que trobeu $\mathrm{i}$ comenteu-les. (2B).

A.12. Heu de distingir solut $\mathrm{j}$ dissolvent en: mistela, aigua de mar, acer, aigua carbònica, cervesa i aire. (2B).

A.13. Prepareu, en tres gots de precipitats, tres dissolucions amb $100 \mathrm{ml}$ d'aigua i 1,4 i $10 \mathrm{~g}$ de sulfat de coure, respectivament. Relacioneu la massa del sulfat afegit amb el volum de líquid. (2B).

A.14. En tres gots de precipitats amb $150 \mathrm{~g} \mathrm{~d}$ d'aigua cadascun, cal dissoldre 5 , 10 i $15 \mathrm{~g}$ de sucre. Relacioneu les masses del solut i del dissolvent. (2B).

A.15. Poseu $3 \mathrm{~g}$ de sal de cuina en $100 \mathrm{ml}$ d'aigua. (al esbrinar: a) el nombre de mols que heu dissolt; b) el nombre de mols de sal necessaris per obteritir un litre d'aigua igual de salada. (3B).

A.16 Heu de fer un esquema que continga totes les formes d'expressar la concentraciód duna dissolució utilitzades abans. (2B/3A).

A.17. En una probeta s'afegeix, a poc a poc, èter a $50 \mathrm{ml}$ d'aigua. Adoneu-vos que, a partir d'un moment, l'èter ja no es dissol. in un altre got de precipitats, s'afegeix alcohol etílic, també a poc a poc, a $50 \mathrm{ml}$ d'aigua. Constateu la diferència que es producix respecte a la dissolució d'èter. (2B).

^.18. Col.loqueu $100 \mathrm{ml}$ d'aigua en un got de precipitats. Afegiu, a poc a poc, petites cullerades de nitrat de potassi fins que no es dissolgui. Calfeu-ho $10^{\circ} \mathrm{C}$ i comproveu que ara es dissol tot. Afegiuhi més nitrat de potassi fins que no es dissolga. Cal repetir l'experiència a diferents temperatures. Després heu de refredar la dissolucio $i$ analitzar el que passa. (2B/3A).

A.19. Fn dissoldre $35 \mathrm{~g}$ de clorur de sodi en $100 \mathrm{mi}$ d'aigua, calfeu fins a l'ebullició i anoteu aquesta temperatura. Cal repetir 1'experiència, però solament amb aigua destil.lada i comparar les dues temperatures obtingudes. Heu d'introduir les hipotesis explicatives cscaients. (3A).

A.20. En un llibre trobem aquestes frases: «La disminució de la temperatura de congelació d'un dissolvent per la presència d'una substància dissolta; s'utilitza per evitar la solidificació de l'aigua de refrigeració en els motors de combustió. Normalment s'empren dissolucions anticongelants constifuides per etanol, etilenglicol i glicerina, que no solidifiquen fins a $-25^{\circ} \mathrm{C}$ ". Comenteu-les per grups. (213).

Aigua (Quantitats expressades en grams per litre)

\begin{tabular}{lllll} 
& molt pura & potable & sospitosa & no potable \\
Clor & $<0.015$ & $<0.040$ & $0.050-0.100$ & $>0.100$ \\
Matèria orgànica & $<0.001$ & $<0.002$ & $0.003-0.004$ & $>0.004$ \\
Nitrats & 0.000 & $<0.015$ & $0.015-0.030$ & $>0.030$ \\
Amoniac Iliure & indicis & $<0.001$ & $0.001-0.002$ & $>0.002$ \\
\hline
\end{tabular}

A.21 Munteu el dispositiu de la figura 2. La paret semipermeable és de paper de cel.lofana. Al recipient $A$ s'introducix una dissolució de glucosa; at B, es posa aigua. Observeu el que ocorre amb l'altura del líquid del zub capil.lar, al llarg del temps. Heu do proposar possibles explicacions. (3A).

Figura 2

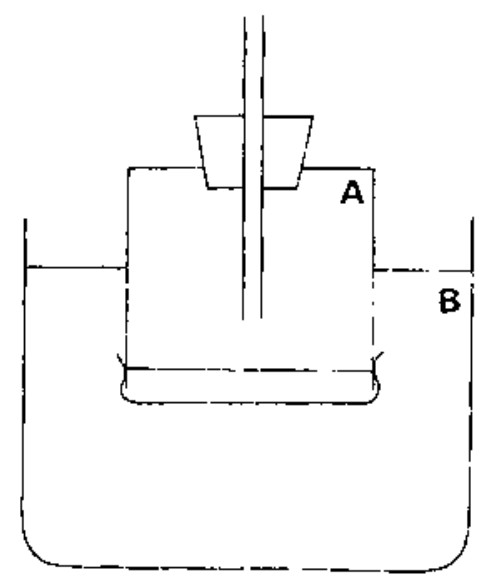

\section{Referencies bibliogràfiques}

Ausubel, P. D., 1978. Psicología Educativa, ('rillas: México).

Carrascosa Alís J., Gil Pérez, D., 1985. La metodologia de la superficialitat $i$ 1'aprenentatge de les Ciències, Enseñanza de las Ciencias, 3(2) pp. 113 120.

Gil Pérez., D., 1982. La investigación en el aula de Físics y Química, (Anaya: Salamanca).

Gil Pérez, D.,1983. Tres paradigmas básicos en la enseñanza de las ciencias, Enseñanza de las' Ciencias 1, pp. 2633.

Gil Pérez, D., Martínez-'Torregrosa, J., 1987. Los programas-guía de actividades: una concreción del modelo constructivista de aprendizaje de las ciencias, Investigación en la escuela, 3, pp. 3-12.

Gimeno Sacristán, J., 1981. Teoria de la enseñanza y desarrollo del currículo. (Anaya: Madrid).

Gutiêrrez, R., 1989. Psicología y aprendizaje de las ciencias. El modelo de Gagné, Enseñanza de las Ciencias, $7(2)$, pp. 147-157.

Piaget, J., 1981. La evolución intelectual entre la adolescencia y la cdad adulta, Infancia y aprendizaje, Monografía Piaget, pp. 13-54.

Piaget, J., Inhelder, B., 1972. De la lógica del niño a la lógica del adolescente, (Paidós: Buenos Aires).

Shayer. M., Adey, P., 1984. La ciencia de enseñar ciencias, (Narcea: Madrid).

\section{IDEAS PREVIAS ERRÓNEAS} ACERCA DEL CONCEPTO DE MASA

Lahore, Alberto A.

Didáctica de la Química. Instituto de Profesores «Artigas".

Montevideo, República Oriental del Uruguay.

Se aplicó una encuesta durante dos años sucesivos -1989 y 1990 . en el curso único de l'ísica y Química para estudiantes de Magisterio, en el Instituto Normal de Montevideo.

(En Uruguay, se ingresa a los Instizutos Normales después de 12 años de enseก̃anza curricular).

l a encuesta tue aplicada después de tratar en clase los conceptos de masa y peso - y no antes-para así acotar la investigación a aquellas ideas erróneas que seguían existiendo en los estudiantes una vez tratados estos temas de forma más o menos tradicional. 
Se encuestaron en total 88 estudiantes, procedentes de 46 institutos de enseñanza secundaria tanto oficiales como privados.

La actividad de laboratorio previa a la encuesta, fue la siguiente:

Calentamiento de cobre en polvo. Medida de la masa del crisol con el contenido, antes y después de 5 minutos de calentamiento. Desputés que los estudiantes midieron la masa final, se les preguntó:

«Cómo interpreta el aumento de masa que tuvo lugar?"

(Está resumida la encuesta, que fue más amplia).

De los diferentes datos recogidos, se destaca el siguiente, relacionado con las ideas previas de los alumnos:

El $31 \%$ del total de encuestados explicaron el atumento de masa atribuyéndolo at aumento de la dureza, solidez, consistencia del material, y explicaciones similares.

Algunos ejemplos:

«Al ponerse más compacto, supuse que iba a ponerse más pesado..."

"Al pasar de polvo a compacto puede influir en el aumento notorio de la masa..."

«Al solidificarse aumenta el peso de la masa..."

«La concentración del poivo en grumos puede cambiar el peso..."

«Su masa es mayor después de solidificado..."

«El calor hace endurecer en forma compacta y aumentar el peso...»

«Por el calor se condensa la masa...»

«Porque queds todo duro, todo comprimido..."

La scmejanza de tantas aftrmaciones no podía ser casual; demostraba que estas ideas erróneas tenian una génesis común.

De acuerdo con el análisis efectuado en cada grupo después de la encuesta, se estimó que el origen de estas creencias era fundamentalmente intuitivo. En las diferentes clases-asamblea, losestudiantes coincidicron en afirmar que «algo con forma definida y compacto, siempre parece intuitivamente que tuviera más peso, más masa, que si está en polvo».

Varios alumnos recordaron también la broma sobre «el kilo de plomo y el kilo de algod6n».

Despucs del tratamiento del tema y antes de la encuesta, los estudiantes sabían dar respuestas correctas sobre los conceptos de masa y peso en cuanto a lo que cada uno de estos conceptos significa oes; por ejemplo, sabían cómo se miden, en qué unidades se expresan, como se vinculan, cómo varían, etc. La encuesta evidenció que muchos estudiantes no sabían lo que estos conceptos «no significan», por ejemplo, que más denso no significa más masivo. Afirmaron con preocupación, que estaban acostumbrados, desde siempre, a una forma de estudio memorística, acrítica, no reflexiva.

La evaluacion fue realizada dos meses después, con preguntas de típo abierto.

Sobre el tema analizado se formuló una pregunta relacionada con las teorías sobre el origen del sistema solar. Para contestarla correctamente, los estudiantes debían razonar acerca de la formación de los dos tipos de planetas: los cercanos al Sol $\rightarrow$ rocosos, metálicos, de alta densidad, poco masivos- y los jupiterianos - de baja densidad y enormemente masivos.

Los resultados de la evaluacion fueron muy satisfactorios.

A la luz de estos resultados, se considera que se debe impartir una enscñanaa "complementada" de los conceptos, en to que respecta a insistir tanto en to que el concepto implica como en lo que no implica, para así corregir exrores que se fueron manteniendo a lo largo de los años en la mente de los alumnos.

Por otra parte, se estima que es siempre importante -se convierte en imprescindibleen la formación de educadores-hacerles conocer a los estudiantes las conclusiones de las investigaciones didácticas sobre el tema que se esté tratando, para lograr que se sientan integrados a un proceso de aprendizaje cooperativo, prioridad fundamental para un aprendizaje constructivista.

\section{Referencias bibliográficas}

Driver, R., 1988. Un enfoque constructivista para el desarrollo del currículo en ciencias, Enseñanza de las Ciencias, (2) pp. 109-120.

García Hourcade y Rodríguez de Ávila, 1988. Ideas previas, esquemas alternativos, cambio conceptual y el trabajo en el aula. Enseñanza de las Ciencias, 6(2) pp. 161-166.

Gil, D. y Carrascosa, J., 1985. Science learning as a conceptual and methodological change, European Journal of Science Education, 7(3), pp. 23I236.
UNA EXPERIENCIA UNIVERSITARIA DE CAMBIO CURRICULAR Y METODOLÓGICO. CURSO DE IN. TRODUCCIÓN A LAS CIENCIAS OUÍMICAS. FUNDAMENTOS EPIS. TEMOLÓGICOS, PSICOLOGYCOS Y DIDÁCTICOS

Aranega, C.P. de, Salinovich, O. e Iparraguirre, L.M.

Colaboración: Leiva, $S$.

Universidad Nacional de Córdoba, República Argentina.

\section{Antecedentes}

En la República Argentina los alumnos ingresan en las Universidades Nacionales dependientes del Estado sin examen de selección previa, con el único requisito de haber finalizado el Nivel Medio de Enseñanza y con títulos que pueden ser de diferentes tipos: bachiller, comercial, técnico profesional (para citar los más corrientes).Los organismos que los otorgan pueden, a su ver, ser nacionales o provinciales, estatales o privados. Si a ello se suman variables de tipo económico, social y cultural, región de procedencia y característica (rural o urbana) de los establecimientos educacionales, se comprenderá el grado de heterogene:dad de Ios ingresantes. Este hecho dificuilta el desarrollo normal de las asignaturas del primer año. Por esta razón, la Facuttad de Ciencias Químicas (Fac. Cs. Qcas) de la Universidad Nacional de Córdoba (UNC) incorporo a su Plan de estudios un Ciclo de Nivelacion consistente en un Curso de Introducción a las Ciencias Químicas, previo al Ciclo Básico Común a las diferentes carreras (durante el cual se desarrolla en cl primer cuatrimestre Física I, Matemáticas I y Química General I).

\section{Propésito del curso}

El proposito general es permitir a los alumnos una más adecuada transición entre el Nivel Medio y el Universitario a través de:

a) Una conexión entre sus conocimientos previos y los que construirá en las asignaturas del Ciclo Básico, una comprensión de la relación entre los conceptos de Química, Física y Matemáticas, el desarrollo de los procesos de pensamiento necesarios para la construcción de nuevos conocimientos, un analisis de la evolución historica de las ciencias y del crecimiento interdependiente de las mismas.

b) El conocimiento del medio universitario en lo que hace a sus aspectos institucionales (académicos, administrativos y políticos) y de la relación entre las dife- 
rentes carreras y la realidad sociocultural del país y la región.

\section{Planificación del curso}

El curso constó de dos partes (A y B) relacionados con los puntos a) y b) expuestos más arriba. En este trabajo expondremos la parte $\mathrm{A}$, que estuvo a cargo de los autores.

\section{Parte del curso}

4.1. Objetivos. Los objetivos directrices fueron: la comprensión de las ciencias (Química, Física y Matemáticas), su contextuación históricosocial e interreiaciones, la construcción de algunos conceptos básicos, el desartollo de procesos cognitivos de análisis y síntesis, razonamiento inductivo, deductivo $y$ analogico, actitudes positivas para la construccion individual y grupal de conocimientos, adquisición de metodologías de estudio.

4.2. Fundamentos epistemológicos, psicologicos y didacticos. Epistemológicamente la asignatura constituyó una unidad de coordinación (Aranega, C.P. de, et al., 1985) que -tomando como eje la Quinica- la relacionaba con la Física y con la Matemática (respetando en cada una de ellas los procesos de construccion conceptuai característicos). Este concepto de coordinación constituye, para algunos autores, una perspectiva importante en el aprendizaje de las ciencias (Barandiarián Piedra, J. 1988).

De cada una de las tres disciplinas cientúficas se seleccionaron algunos conceptos estructurantes (Gagliardi, R. 1986, Gagliardi, R. y Giordan 1986). La orga. nización de los conceptos se evidencia en el diagrama 1.

Otra característica del curso fue -como se dijo- la introducción de la historia de las Ciencias, considerada hoy esencial por diversos autores (Cawthorn y Rowell 1978, Giordan 1985, Izquierdo 1988. Hodson 1985, Saltier y Viennot 1985, Yager y Penick 1978).

El fundamento psicológico lo constituyeron las teorías constructivistas del aprendizaje (Driver, R. 1981, 1986, 1988, Driver, R. y Easley, J. 1978, Posner, G.J., Strike, K.A., Hewson, P.W. y Gertzog, W.A. 1982, Gilbert, J.K. y Watts, D.M. 1983, Hewson, M.G. y Hewson, P.V. 1983, Osborne, R.J. y Wittrock, M. I98S).

En palabras de Gagliardi (1986) «la idea de concepto estructurante solo tiene sentido en una concepción constructivista». A la luz de estas concepciones se requiere partir de las ideas intuitivas de los estudiantes (Driver 1986) o, lo que es lo mismo, de sus esquemas conceptuales

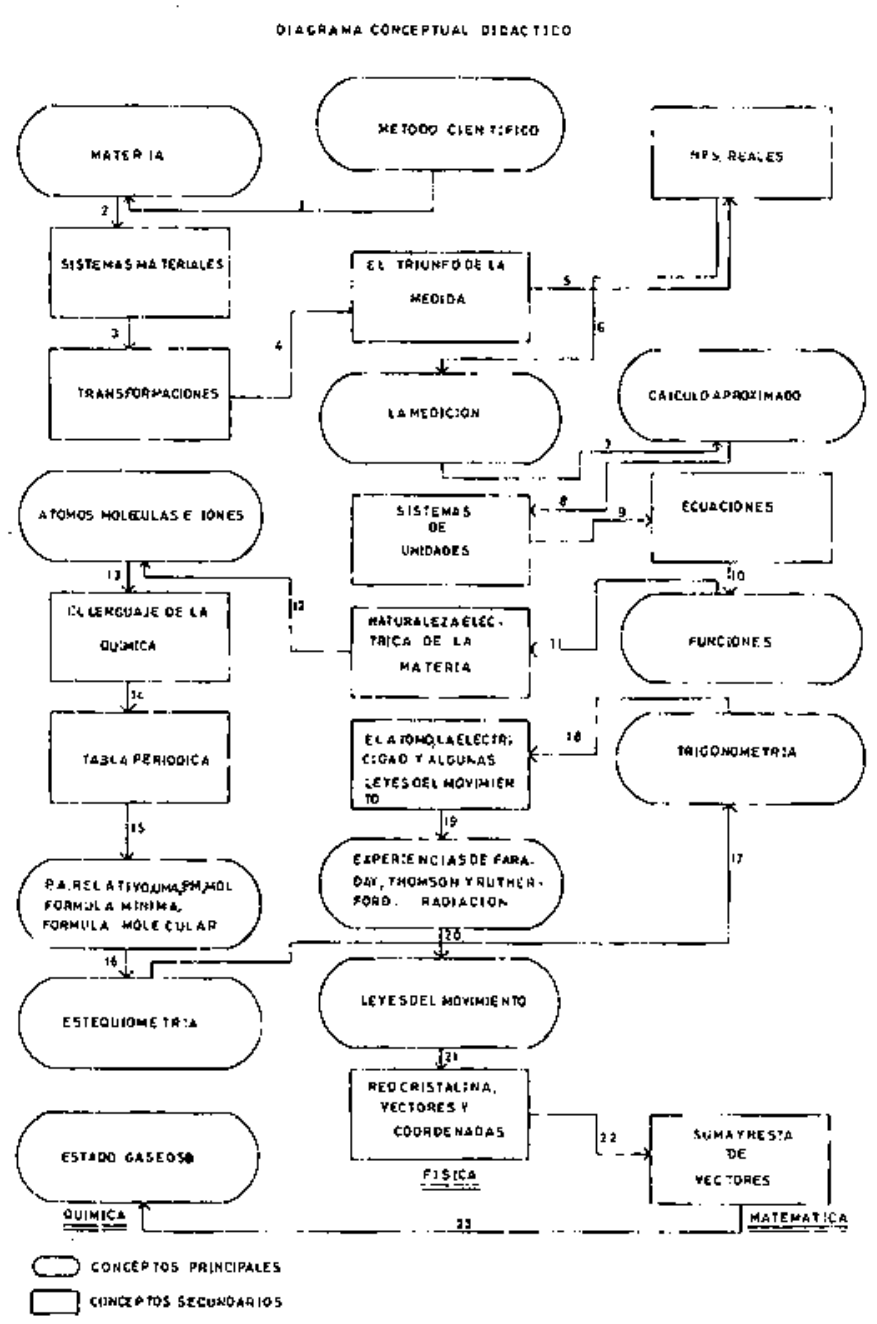

alternativos (Driver y Easley 1978), de sus preconceptos (Clement y Brown 1983), de sus teorías ingenuas (Caramazza et al. 1981) y producir en esos alumnos -a partir de un nuevo aprendizaje- un cambio conceptual (Gil Pérez y Carrascosa 1985), que les permita explicar y resolver situaciones que antes de dicho cambio no podfa realizar (Posner et al. 1982).

Desde el punto de vista didactico el paradigma adoptado fue el de «descubrimiento dirigido o guiado" (Gil Pérez, D. $1983,1986)$ que se propone un cambio conceptual, metodológico y didáctico por parte de los alumnos.

A la luz de dicho paradjgma se selecionaron las actividades adecuadas y se las organizó acorde con las sugerencias de Driver (Driver, R. 1986).

4,2.1. Estrategia didáctica. La estrategia didáctica utilizada fue el estudio au- tónomo: individual y grupal (pequeño grupo y grupo grande o comisión completa). Es decir, se combinaron técnicas de trabajo individual y grupal.

4.2.2. Recurso didáctico. Poder desarroLlar una estrategia como la planteada en un curso introductorio masivo (superior a los 1000 alumnos), donde además de la heterogeneidad estudiantil existía una heterogeneidad en el cuerpo docente, nos condujo a utilizar una estrategia didáctica específica: las guías de estudio.

Las «guías» fueron desarrolladas en su mayoría por docentes del curso, apoyándonos para ello en la sugerencia de otros autores (Driver, R. y Oidham, V. 1986).

4.3. Personal docente. El curso estruvo a cargo de 27 docentes (alumnos de los 2 últimos años de las diferentes carreras de la facultad, graduados y ayudantes alumnos de los diferentes departamentos, todos 
fucron seleccionados, previo examen. Cada uno tuvo a cargo comisiones de entre 20 y 35 estudiantes. Su función era la de coordinar las actividades de los alumnos previstas en las guías. Cada tumo (T1, $\mathrm{T} 2, \mathrm{~T} 3$ ) tenía un supervisor y un subsupervisor, responsable de las actividades académicas y administrativas de entre 12 y 15 comisiones. Éstos eran Jefes de Trabajos Prácticos de la Facultad, la mayoría de clios inscriptos en la carrera de doctorato o finalizada la misma. Todos los docentes participaron en reuniones preparatorias para familiarizarse con la metología de trabajo.

4.4. Duración. Se inicio el 16 de enero y finalizo el 10 de marzo de 1989 .

4.5. Sistemas de evaluación. Se realizaron: (a) evaluaciones diagnósticas (con el objeto de detectar y resolver problemas de aprendizaje y brindar a los estudiantes elementos de autoevaluación, b) evaluaciones parciales de los productos de aprendizaje (éstas cumplimentaban el requisito de aprobación del curso y se realizaron dos: al finalizar la $i^{3}$ y la $2^{5}$ parte del curso). c) evaluación final de producto (se utilizaba con los alumnos que no hubiesen aprobado las evaluaciones parciales y tenía carácter de evaluación recuperatoria integrada).

Las evaluaciones de tipo b) y c) cran pruebas objetivas de 40 ftems de opción múltiple con cuatro alternativas.

Tabla 1

Medianas y diferencias de las medianas de las notas obtenidas por los diferentes turnos en el primer parcial.

\begin{tabular}{ll|l|l|l|l|l} 
& \multicolumn{1}{c}{$\mathrm{T} 1$} & $\mathrm{~T} 2$ & $\mathrm{~T} 3$ & $\mathrm{~T} 1-\mathrm{T} 2$ & $\mathrm{~T} 1-\mathrm{T} 3$ & $\mathrm{~T} 2-\mathrm{T} 3$ \\
\hline $\mathrm{N}$ & 478 & 299 & 282 & 777 & 760 & 581 \\
$\mathrm{Md}$ & 5,23 & 4,82 & 4,29 & 5,06 & 4,85 & 4,56 \\
$\mathrm{X}$ & & & & 7,50 & 45,28 & 14,93 \\
& & & & & $*$ & $*$ \\
\hline
\end{tabular}

"Significativo al nirel de $=.01$

\section{Tabia II}

Medianas y diferencias de las media-

nas de las notas obtenidas por los ulumnos de los diferentes turnos en el segundo parcial.

\begin{tabular}{l|l|l|l|l|l|l} 
& $\mathrm{T} 1$ & $\mathrm{~T} 2$ & $\mathrm{~T} 3$ & $\mathrm{~T} 1 . \mathrm{T} 2$ & $\mathrm{~T} 1-\mathrm{T} 3$ & $\mathrm{~T} 2-\mathrm{T} 3$ \\
\hline $\mathrm{N}$ & 457 & 290 & 265 & 747 & 722 & 555 \\
$\mathrm{Md}$ & 5,84 & 4,18 & 3,82 & 5,23 & 5,09 & 3,97 \\
$\mathrm{X} 2$ & & & $\begin{array}{l}54,49 \\
*\end{array}$ & $\begin{array}{l}88,36 \\
*\end{array}$ & $\begin{array}{l}7,25 \\
*\end{array}$ \\
\hline
\end{tabular}

4.6. Resultados. En las tablas $n^{3}$ I y II se presenta la mediana (Md) de las notas obtenidas por los alumnos en la $I^{2}$ y $2^{\text {at }}$ evaluación parcial (1P y $2 \mathrm{P}$ ) y la prueba de diferencia de mediana ( $\mathrm{X}^{2}$. chi cuadrado con corrección de Yates) para determinar el grado de significación de las diferencias de la puntuación obtenida por los alumnos de tos tres turnos (T1, T2 y T3). En ningún caso las diferencias encontradas fueron significativas a un nivel de $=05$

En el gráfico I se presenta el porcentaje de alumnos aprobados y no aprobados de los presentados a la evaluación recuperatoria.

\section{Gráfico 1}

Porcentaje de alumnos ausentes, aprobados y no aprobados en el recuperatorio.

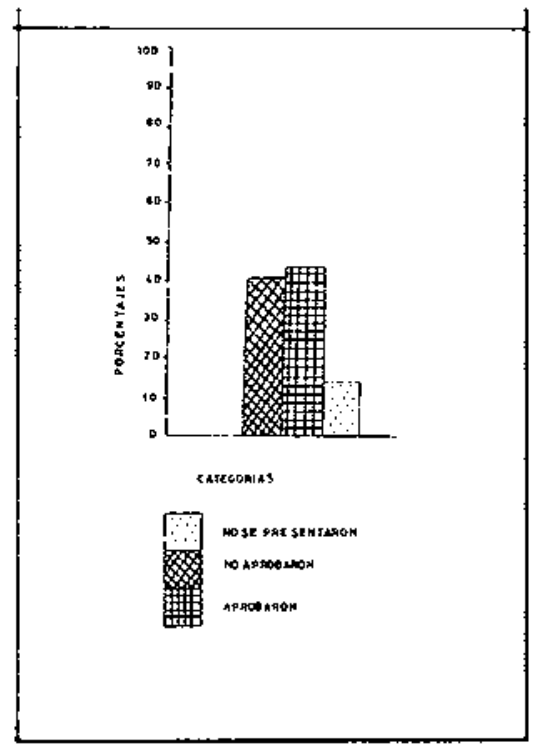

Del totat de 1339 alumnos, un $12 \%$ (261) no asistió nunca De los 1078 que lo hicieron, un $5 \%$ (50) desertaron después del $1 P$, la mayoría habiéndolo aprobado. El $62 \%$ (672) aprob6 el curso por el sistcma de los 2 parciales y el $15 \%$ (158) por la evaluación recuperatoria, con lo que ascendió el número de aprobados a un $77 \%$. El total de altumnos que no aprobó (quedando en la categoría de to que llamamos alumno regular fue de $17 \%$ ( 47 reprobados en el recuperatorio y 51 que no se presentaron (tablas II y III y gráfico 2). Las diferencias de proporciones entre los alumnos desertores, aprobados y regularizados fueron significativas a nivel de $=0$.

4.7. Evaluación del curso por parte de los docentes. Se confecciono una encuesta que se entreg 6 a los 27 docentes del curso siendo respondida de forma voluntaria por 13 (casi la mitad). Las preguntas se
Gráficico 2

Porcentaje de alumnos desertores aprobados (por el sistema de parciales o por el recuperatorio) y de regularizados.

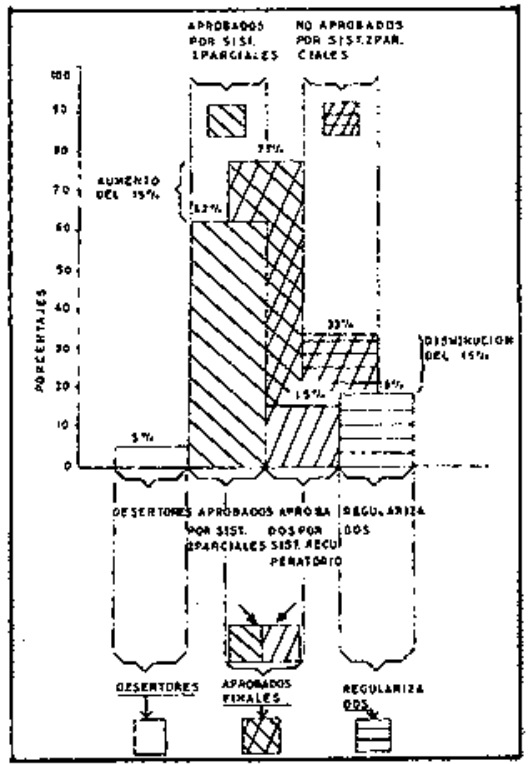

relacionaban con el grado de adecuación de los contenidos seleccionados y su organización, de las actividades de aprendizaje propuestas a los alumnos y de las evaluaciones y su dificultad. Los resultados obtenidos en la escala de adecuación fueron ampliamente positivos. 'También se les preguntó acerca del enfoque epistemológico de coordinación de asignaturas, fue buena para el $62 \%$, regular para ct $38 \%$, mala para ninguno. La metodología utilizada fue a juicio del $100 \%$ mejor que la tradicionalmente empleada. El 100\% de los docentes emitió un juicio muy positivo como síntesis del curso.

\section{Conclusiones}

Del análisis de los resultados de la parte A del curso se desprende que los objetiyos directrices se Iograron (fue clevado el número de alumnos aprobados cont respecto al do no aprobados, sirvió además como elemento orientador temprano para quienes desertaron, un porcentaje muy bajo). Este hecho y al juicio de los docentes sugiere que los contenidos y actividades de aprendizaje seleccionados empleando determinadas estrategias y recursos didácticos resultó adecuado, como así también las evaluaciones utilizadas con fines de diagnóstico y de resulrados parciales.

Si a esto se agrega el informe correspon diente al coordinador de la parte $\mathrm{B}$ (n) presentado en este trabajo) se puede concluir que el propósito para el cual se desarrollo el curso fue cumplimentado. 
Tabla III

Número y porcentaje de alumnos desertores, aprobados (por sistema de dos parciales y por el sistema de recuperación y de reguiarizados).

\begin{tabular}{|ccccc|}
\hline Categoría & Nímero & Porceniaje $\%$ & Número & Porcentaje \% \\
\hline $\begin{array}{c}\text { Desertores } \\
\text { por el sistcma } \\
\text { de dos parciales }\end{array}$ & 50 & 5 & 50 & 5 \\
$\begin{array}{c}\text { Aprobados } \\
\text { por el sistema } \\
\text { recuperatorio }\end{array}$ & 672 & 62 & 830 & 77 \\
$\begin{array}{c}\text { Reguiarizados } \\
\text { Total }\end{array}$ & 158 & 15 & 198 & 18 \\
\hline
\end{tabular}

Los reajustes que al mismo se realizaron a partir de esta experiencia permitieron una segunda versión en 1990 , sobre la base de la cual se implementará en 1991 una tercera versión. De ellas se concluirá acerca de la validez del marco teórico (fundamentos epistemológicos, psicologícos y didácticos) que le sirvió de apoyo.

\section{Referencias bibliográficas}

Aranega, C.P. de, 1986. Un marco térico referencial para la didáctica: teorías psicológicas contemporáneas y actuales del aprendizaje, Trabajos de Educación en Ciencias, Univ. Nac. de Cordoba, Córdoba, Vol. 2, pp. 1-132.

Aranega, C.P. de, Barmaat, M.L.R. de, De Longhhi, A.L. Coordinación, combinación e integración de disciplinas en el nivel medio de enseñanza, Trabajos de Educación en Ciencias. Univ. Nac. de Córdoba, Córdoba, Vol. 1,pp. 1-77.

Aranega, C.P. de, De Longhi, A.L., 1987. Selección y organización de contentdos en la enseñanza aprendizaje de la ciencia y la tecnologia, Trabajos de Educación en Ciencias, Univ. Nac. de Córdoba, Córdoba, Vol. 3, pp. 1-36.

Barandarián Piedra, J., 1988. El modelo de enseñanza-aprendizaje de las ciencias en la reforma de las enseñanzas medias, Enseñanza de las Ciencias, Vol. 6 (2), pp. 167-170.

Caramazza, A., McCloskey, M. y Green, B., 1981. Naive Beliefs in sophisticated subjects, misconceptions about trajectories of objects, Cognitive, Vol. 9 , pp. 117-123.
Cawthom, E.R. y Rowell, J.A., 1978. Epistemology and Science education, Studies in science education, Vol. 59 (3), pp. 423-430.

Chalmer, A.F., 1982. ¿Qué es esa cosa llamada Ciencia? (Siglo XXI: Madrid).

Clement, J. y Brown, D., 1983. Using analogica resasoning to deal with "deep» misconceptions in physics, Working paper University of Massachusset. (Amherst, M.A.)

Coll, C., 1987. Psicología y curriculum (Laia. Barcelona).

Driver, R., 1981. Pupils alternative frameworks in science, European Journal of Science Education, Vol. 3 (1), pp. 83-101

Driver, R., 1986. Psicología congnoscitiva y esquemas altermativos de los alumnos, Ensiñanza de las Ciencias, Vol. 4 (1), pp. 3-15.

Driver, R., 1988. Un enfoque constructivista para el desarrotio del currículum en ciencias, Enseñanza de las Ciencias, Vol. 6 (2), pp. 109-120.

Driver, R. y Easley, J., 1978. Pupils and paradigms: it review of literature related to concept development in adolescent science students, Studies in Science Education, Vol S. pp. 6I-84.

Driver, R. y Oldham, V., 1986. A constructivist approach to curriculum development in science, Studies in Science Education, Vol. 13, pp. 105122.

Gagliardi, R. y Giordan, A., 1986. La historia de las Ciencias: una herramicnta para la enseñanza, Enseñanza de las Ciencias, vol. 4 (3), pp. 253258 .
Gagliardi, R., 1988. Cómo utilizar la historia de las ciencias en la enseñanza de las ciencias, Enseñanza de las Ciencias, Vol. 6 (3), pp. 291-296.

Gil Pérec, D., 1983. Tres paradigmas básicos en la enseñanza de las ciencias, Enseñanza de las Ciencias, Vol. 1 (1), pp. 26-33.

Gil Pérez, D., 1986. I a metodología científica y la enseñanza de las ciencias, unas relaciones controvertidas, Enseñanza de las Ciencias, Vol. 4(2), pp 11-121

Gil Pérez, D. y Carrascosa, J., 1985. Science learning as a conceptual methodological change, European Journal of Science Education, Vol. 7 (3), pp. 231-236.

Gilbert, J.K. y Watts, D.M., 1983. Concepts, misconceptions and aiternative conceptions changing perspective in science education. Studies in Science Eaducation, Vol. 10, pp. 61-98.

Gimeno Sacristán, J y Pérez Gómez, A., 1985. La enseñanza: su teoría y su práctica. (Akal, Madrid).

Hewson, M.G. y Hewson, P.V., 1983. Effects of instruction ussing student's prior knowledge and conceptual change strategies on science learning, Journal of Research in Science Teaching.

Hodson, D., 1985. Philosophy of Science, science and science education, Studies in Science Education, Vol. 12 , pp. 25-57.

Izquierdo, M., 1988. I. a contribució de la teoria del flogiste a l'estructuració actual de la ciencia química, Enseñanza de las Ciencias, Vol. 6(1), pp. 67-74.

Osborne, R. y Wittrock, M., 1985. The generative learning model and iss implications for Science education. Studies in Science Education. Vol. 12, pp. 59-87.

Posner, J., Strike, K. A., Hewson, P.W. y Gertzow, W.A., 1982. Accommodation of a scientific conception: towards a theory of conceptual change, Science Education, Vol. 66, pp. 211-227.

Saltier, E. y Viennot, I.., 1985. ¿Qué aprendemos de las semejanzas entre las ideas históricas y el razonamiento espontáneo de los estudiantes?, Enseñanza de las Ciencias, Vol. 3 (2), pp. 137-144.

Yager, R.E. y Penick, J.E., 1983. Analysis of the current problems with school science in the USA, European Journal of Science Education, Vol. 5, pp. 463-459. 